\title{
Uso de interfaces cérebro-computador em crianças com TDAH: Uma revisão
}

\section{sistemática}

\author{
Use of brain-computer interfaces in children with ADHD: A systematic review \\ Uso de interfaces cerebro-computadora en niños con TDAH: Una revisión sistemática
}

Recebido: 07/06/2021 | Revisado: 13/06/2021 | Aceito: 16/06/2021 | Publicado: 30/06/2021

Nayara Magda Gomes Barbosa da Costa

ORCID: https://orcid.org/0000-0002-9162-5182 Universidade Federal do Ceará, Brasil

E-mail: nayaramenfer@hotmail.com

Edgar Marçal

ORCID: https://orcid.org/0000-0001-5037-2724 Universidade Federal do Ceará, Brasil E-mail: edgar@virtual.ufc.br

Meline Mesquita de Carvalho

ORCID: https://orcid.org/0000-0003-2959-6322 Universidade Federal do Ceará, Brasil E-mail: meline@virtual.ufc.br

Tiago da Costa Silva Barbosa

ORCID: https://orcid.org/0000-0002-4347-3006

Instituto Federal de Educação, Ciência e Tecnologia do Piauí, Brasil E-mail: tiago.silva@ifpi.edu.br

\begin{abstract}
Resumo
O transtorno de déficit de atenção/hiperatividade (TDAH) tem início na infância e apresenta como sintomas mais comuns a falta de atenção, hiperatividade e dificuldade de autocontrole. As Interfaces Cérebro-Computador (ICCs) podem auxiliar no tratamento de pacientes com TDAH. Nesse contexto, o presente artigo trata-se de uma revisão sistemática que objetivou-se em relatar como as ICCs estão sendo utilizadas no tratamento de crianças com TDAH, baseado na hipótese de que a utilização das ICCs corrobora com a melhora do tratamento dessas crianças. Para isso, realizou-se uma análise bibliográfica nas publicações científicas presentes nas bibliotecas digitais IEEE Xplore, PubMed e ScienceDirect. Na análise foram consideradas as publicações dos anos de 2015 a 2020, no idioma inglês. Verificou-se que diferentes hardwares foram utilizados no tratamento de crianças com TDAH por meio das ICCs, no geral, associados a jogos digitais interativos. As ICCs utilizadas desempenharam um importante papel, podendo-se considerar que as mesmas corroboraram para melhorias na atenção, habilidades sociais e comportamentais, bem como, na reorganização da rede funcional do cérebro.
\end{abstract}

Palavras-chave: Interface cérebro-computador; Saúde mental; Eletroencefalograma.

\begin{abstract}
Attention deficit / hyperactivity disorder (ADHD) starts in childhood and presents as the most common symptoms the lack of attention, hyperactivity and difficulty in self-control. Brain-Computer Interfaces (BCIs) can assist in the treatment of patients with ADHD. In this context, this article is a systematic review that aimed to report how the BCI are being used in the treatment of children with ADHD based on the hypothesis that the use of BCIs corroborates with the improving the treatment of these children. For this, a bibliographic analysis was carried out in the scientific publications present in the digital libraries IEEE Xplore, PubMed and ScienceDirect. In the analysis, publications from 2015 to 2020 in english were considered. Differents hardwares were used to treat children with ADHD through BCIs in general associated with interactive digital games. The BCIs used played an important role being able to consider that they corroborated for improvements in the attention, social and behavioral skills, as well as, in reorganizing the functional brain network.
\end{abstract}

Keywords: Brain-computer interface; Mental health; Electroencephalogram.

\section{Resumen}

El trastorno por déficit de atención con hiperactividad (TDAH) comienza en la niñez y los síntomas más comunes son falta de atención, hiperactividad y dificultad en el autocontrol. Las Interfaces Cerebro-Computadora (ICC) pueden ayudar en el tratamiento de pacientes con TDAH. En este contexto, este artículo es una revisión sistemática tenido como objetivo informar cómo se utilizan las ICC en el tratamiento de niños con TDAH, basado en la hipótesis de que el uso de ICC corrobora con la mejora en el tratamiento de estos niños. Para ello, se realizó un análisis bibliográfico en publicaciones científicas presentes en las bibliotecas digitales IEEE Xplore, PubMed y ScienceDirect. En el 
análisis se consideraron publicaciones de los años 2015 a 2020, en inglés. Se encontró que en el tratamiento de niños con TDAH a través de ICC se utilizaron diferentes hardware, en general, asociados a juegos digitales interactivos. Los ICC utilizados jugaron un papel importante y se puede considerar que contribuyeron para mejoras de las habilidades de atención, sociales y conductuales, así como a la reorganización de la red funcional del cerebro.

Palabras clave: Interfaz cerebro-computadora; Salud mental; Electroencefalograma.

\section{Introdução}

O déficit de atenção/hiperatividade (TDAH) é um transtorno comum na infância que segundo Silva (2014) apresentase basicamente por três sintomas: desatenção, impulsividade e hiperatividade física e mental. O TDAH acarreta alterações nas funções executivas que, conforme Munakata, Michaelson, Barker e Chevalier (2013), podem ser compreendidas como um conjunto de processos cognitivos que dão suporte à regulação dos pensamentos, emoções e comportamentos. O cérebro possui inúmeras funções cognitivas dentre as quais destacam-se pela sua relevância: focalizar a atenção, compilar e guardar conhecimentos. A atenção é considerada como o eixo central das aptidões cognitivas (Jiang, Abiri \& Zhao, 2017). Segundo a American Psychiatric Association (2014), 5\% das crianças são diagnosticadas com TDAH. Embora acometidas por esse transtorno, essas crianças possuem traços de criatividade, são amáveis, talentosas, gostam de inovar e aplicar suas inovações, conforme Rief (2005).

Quando não existe um diagnóstico prévio e um tratamento direcionado, o TDAH pode ocasionar graves consequências na qualidade de vida do paciente e de seus familiares. Segundo Abrahão e Fantacini (2017) por influenciar no comportamento e consequentemente no processo ensino-aprendizagem, o TDAH pode gerar conflitos pessoais, familiares e escolares. Exames de imagens e o Eletroencefalograma (EEG) podem ser utilizados no diagnóstico de pacientes com TDAH. De acordo com Dickstein, Bannon, Castellanos e Milham (2016) as crianças acometidas com esse transtorno apresentam nos exames de imagens regiões do cérebro afetadas com uma diminuição da atividade do lobo frontal repercutindo no córtex anterior cingulado, córtex pré-frontal dorsolateral, córtex pré-frontal inferior e córtex orbitofrontal, parcialmente nos gânglios basais, tálamo e córtex parietal. Snyder e Hall (2006) observaram que nos exames de EEG as crianças com TDAH apresentaram um ampliamento das ondas teta e decréscimo nas ondas beta.

Quanto aos recursos terapêuticos, após diagnosticados, os portadores de TDAH podem ser submetidos a tratamentos medicamentosos, terapias comportamentais ou psicossociais e terapia neurofeedback (Cortese \& Coghill, 2018; Boland et al., 2020). Os medicamentos quando utilizados têm benefícios nas catecolaminas, o que garante melhoria nos aspectos de atenção. Porém podem apresentar diversos efeitos colaterais, o que dificulta a manutenção do tratamento. As terapias comportamentais ou psicossociais melhoram a sociabilidade, mas, para que seus efeitos sejam mais expressivos, é necessário que o paciente participe por um longo período de tempo (Kessler et al., 2006; Hechtman et al., 2016). A terapia neurofeedback configura-se como um procedimento não invasivo e que não faz uso de medicações, fundamentado nas ICCs, tendo a mesma apresentado respostas exitosas.

No tratamento via neurofeedback a atividade cerebral do paciente é medida por meio do EEG, sendo essa processada e calculada ao vivo. Após análises nos dados do EEG, o sistema emite um novo sinal ao paciente como resposta ao seu comportamento inicial. Dessa forma o feedback faz com que o paciente tenha o autocontrole das suas funções cerebrais nas partes que envolvem o cognitivo e o comportamento (Geppert, Huster \& Herrmann, 2017). Para Guan et al., (2020), as ICCs têm auxiliado no tratamento do TDAH, melhorando a cognição cerebral no domínio da atenção.

Com base no que foi exposto, o presente artigo tem como objetivo identificar os principais benefícios, tecnologias, softwares, técnicas e desafios proporcionados pelo uso das ICCs no tratamento de crianças com TDAH. Quanto a estrutura, o restante do artigo está organizado em quatro seções que abrangem respectivamente: a metodologia pormenorizada da revisão sistemática, os resultados relevantes obtidos ao longo do estudo, a discussão das principais contribuições da revisão sistemática 
baseando-se nas questões de pesquisa e as considerações finais.

\section{Metodologia}

O artigo trata-se de uma pesquisa bibliográfica na qual o método escolhido para análise do estado da arte foi a Revisão Sistemática de Literatura proposta por Kitchenham (2004). Para Marçal, Kubrusly e Silva (2017) a técnica adotada em Kitchenham (2004) permite agrupar e avaliar as evidências empíricas de um determinado campo de estudo a partir da análise das pesquisas relevantes disponíveis no assunto de interesse, obtendo-se assim conclusões sobre as questões de pesquisa definidas.

A revisão foi sequenciada nas seguintes etapas de acordo com Kitchenham (2004):

(i) Planejamento - que compreendeu a definição das informações do protocolo de revisão, tais como: questões de pesquisa, string de busca e bases de artigosutilizadas na pesquisa;

(ii) Condução - que consistiu na aplicação da string de busca nas diferentes bases de pesquisa, seguido da filtragem dos artigos com base nos critérios de inclusão e posterior extração e sintetização dos dados dos artigosselecionados;

(iii) Relato - representado pela comunicação dos resultados da revisão, por meio da publicação em artigo científico, relatórios técnicos ou em sessão detrabalhos acadêmicos de dissertação de mestrado ou de tese de doutorado. Para relatar como as ICCs estão sendo empregadas no tratamento de crianças com TDAH, utilizou-se questões de pesquisas abrangentes que envolviam aspectos metodológicos e aspectos técnicos de maior relevância. Nesse sentido, buscou-se elaboraruma pesquisa bibliográfica que possibilitasse uma visão amplificada sobre a aplicabilidadedas ICCs nas intervenções em crianças com TDAH. As questões de pesquisa e suas motivações estão presentes no Quadro 1.

Quadro 1. Questões de pesquisa.

\begin{tabular}{|l|ll|}
\hline \multicolumn{1}{|c|}{ Questão de Pesquisa } & \multicolumn{1}{|c|}{ Motivação } \\
\hline $\begin{array}{l}\text { QP1- Quais os benefícios e ganhos em se utilizar interfaces } \\
\text { cérebro-computador no tratamento decrianças com TDAH? }\end{array}$ & $\begin{array}{l}\text { Verificar se o uso de interfaces cérebro- } \\
\text { computador colabora com o tratamento de crianças } \\
\text { com TDAH. }\end{array}$ \\
\hline $\begin{array}{l}\text { QP2- Quais os hardwares que estão sendo utilizados para } \\
\text { auxiliar no tratamento de crianças com TDAH através de } \\
\text { interfaces cérebro-computador? }\end{array}$ & $\begin{array}{l}\text { Identificar quais os equipamentos que estão sendo } \\
\text { utilizadas para auxiliar no tratamento de crianças com } \\
\text { TDAH por meio de interfaces cérebro-computador. }\end{array}$ \\
\hline $\begin{array}{l}\text { QP3- Quais os softwares que estão sendo utilizados para } \\
\text { auxiliar no tratamento de crianças com TDAH através de } \\
\text { interfaces cérebro-computador? }\end{array}$ & $\begin{array}{l}\text { Detectar quais os programas utilizados no auxílio do } \\
\text { tratamento de crianças com TDAH por meio de } \\
\text { interfaces cérebro-computador. }\end{array}$ \\
\hline $\begin{array}{l}\text { QP4- Quais os procedimentos que estão sendo utilizadas } \\
\text { para auxiliar no tratamento de crianças com TDAH através } \\
\text { de interfaces cérebro-computador? }\end{array}$ & $\begin{array}{l}\text { Descrever os procedimentos que estão sendo } \\
\text { utilizados no tratamento de crianças com TDAH por } \\
\text { meio de interfaces cérebro-computador. }\end{array}$ \\
\hline $\begin{array}{l}\text { QP5- Quais os desafios, limitações e problemas para se } \\
\text { utilizar interfaces cérebro-computador no tratamento de } \\
\text { crianças com TDAH? }\end{array}$ & $\begin{array}{l}\text { Relatar as principais dificuldades para se utilizar } \\
\text { interfaces cérebro-computador no tratamento de } \\
\text { crianças com TDAH. }\end{array}$ \\
\hline
\end{tabular}

Fonte: Autores. 
Definiu-se a string de busca de modo que a mesma fornecesse uma ampla cobertura com tamanho razoável, conforme Marçal, Kubrusly e Silva (2017). Para a busca da bibliografia foram utilizadas as seguintes bibliotecas digitais de artigos de fontes relevantesnas áreas de engenharia de software e saúde: IEEE-Xplore, PubMed, Science Direct, SciELO e Periódicos Capes. Os termos contemplados na string de busca basearam-se nas questões de pesquisa, sendo os mesmos agrupados nos escopos: saúde mental e tecnologia. O booleano AND foi usado para juntar os termos. A string de busca ADHD AND Brain Computer Interface foram utilizadas nas bibliotecas IEEE-Xplore, PubMed e Science Direct, enquanto TDAH AND Interface Cérebro Computador na SciELO e Periódicos Capes. A string de busca está presente no Quadro 2.

Quadro 2. String de busca.

\begin{tabular}{|l|l|l|}
\hline Escopo & String & String \\
\hline Saúde Mental & $($ ADHD) AND & (TDAH) AND \\
\hline Tecnologia & (brain computer interface) & (Interface Cérebero Computador) \\
\hline
\end{tabular}

Fonte: Autores.

Após a busca inicial, foi realizada uma verificação nos artigos selecionados observando se os mesmos se adequavam às questões de pesquisa. Para a verificação foram estabelecidos os critérios que os artigos deveriam atender, sendo incluídos aqueles que apresentassem: a descrição do estudo de caso, que obrigatoriamente deveria envolver tratamento em crianças com TDAH; e, o procedimento que está sendo utilizado na ICC. Além dos critérios de inclusão, nas bibliotecas pesquisadas foram utilizados os filtros: ano de publicação, sendo considerados os artigos publicados entre 2015 e 2020; e, idioma, considerandose somente os artigos publicados em inglês e português.

\section{Resultados}

A Figura 1 apresenta o resultado da quantidade de estudos encontrados ao longo das fases de seleção do material bibliográfico. O levantamento inicial resultou em 73 artigos, porém após aplicação das fases de filtragem somente 7 foram considerados aptos para o presente estudo. Na primeira fase da pesquisa foi utilizada a string de busca nas bibliotecas digitais selecionadas o que resultou em um total de 73 artigos. Posteriormente, na fase 2, foram aplicados os critérios de filtragem de acordo com cada biblioteca obtendo-se assim 51 artigos. Na fase 3 foram aplicados os critérios de inclusão nos títulos e resumos dos 51 artigos selecionados na fase anterior, reduzindo o total para 41 artigos. Por fim, na fase 4 foi realizada a leitura dos artigos na íntegra aplicando os critérios de inclusão em todo o artigo, resultando em 7 estudos finais. As seções posteriores descrevem os resultados das análises dos 7 artigos considerando as questões de pesquisa. 
Figura 1. Quantidade de artigos obtidos nas fases de revisão sistemática.

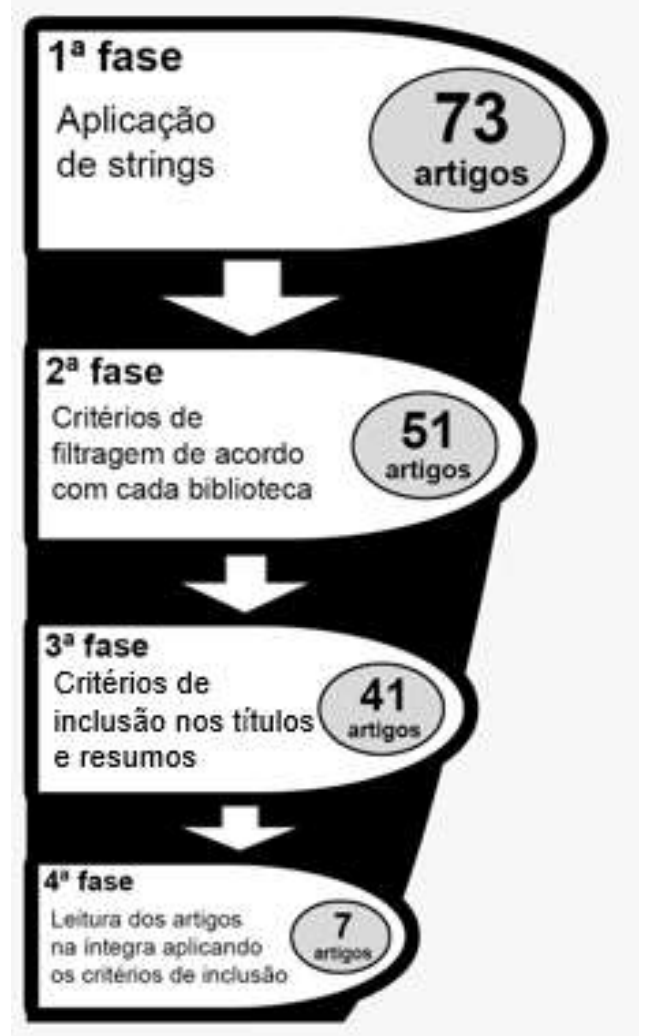

Fonte: Autores.

3.1 QP1. Quais os benefícios e ganhos em se utilizar interfaces cérebro-computador no tratamento de crianças com TDAH?

Analisando os artigos finais, observou-se que os benefícios e ganhos relacionados ao uso das ICCs no tratamento em crianças com TDAH foram:

- Reorganiza a rede funcional do cérebro das configurações mais regulares para as mais aleatórias;

- Melhora no foco e na concentração mental, das crianças em realizar as atividades diárias;

- Resultado positivo bastante exitoso na atenção, as crianças mostraram melhorias as quais foram relatadas pelos pais e a equipe médica;

- Ganho nas habilidades sociais e o controle comportamental obtiveram melhoras, resultando em uma melhor integração;

- Redução dos sintomas de internalização, que secundariamente tem melhoras no TDAH;

- Melhora na conectividade funcional que diminuiu dentro da rede de atenção ventral e entre as redes de tarefa positiva e as regiões subcorticais.

3.2 QP2. Quais os hardwares que estão sendo utilizados para auxiliar no tratamento de crianças com TDAH através de interfaces cérebro-computador?

Quanto aos hardwares, analisando os artigos finais, observou-se que para auxiliar no tratamento das crianças com TDAH por meio das ICCs foram utilizados: head-band com sensores secos (Lim et al., 2019; Qian et al., 2018; Blandón, Muñoz, Lopez \& Gallo, 2016); aparelho de ressonância magnética (fMRI) (Qian et al., 2018; Guan et al., 2020); Headset Mindwave Mobile (Faseeha, Naseem, Saleem, Jahan \& Jamil, 2018; Park, Kihl, Park, Kim \& Chang, 2016); placa Arduino 
Leonardo ATmega32u4 (Faseeha et al., 2018); controlador Kinect da Microsoft (Park et al., 2016); robô Sanbot Elf Humanoide (Arpaia, Duraccio, Moccaldi \& Rossi, 2020); óculos de estimulação Moverio BT-200 AR (Arpaia et al., 2020); computador com placa única Raspberry Pi 3 e conversor analógico-digital Olimex EEG-SMT (Arpaia et al., 2020).

\subsection{QP3. Quais os softwares que estão sendo utilizados para auxiliar no tratamento de crianças com TDAH através de interfaces cérebro-computador?}

Em relação aos softwares auxiliares no tratamento de crianças com TDAH por meiodas ICCs os estudos analisados utilizaram: jogo Cogoland, que consiste no controle de um personagem para a realização das atividades propostas, cuja velocidade de realização depende da atenção do jogador (Lim et al., 2019; Qian et al., 2018); jogo digital para o treinamento comportamental por narrativas, no qual cada participante é integrante da história e determina o final da mesma, o funcionamento do jogo requer atenção do participante (Park et al., 2016); diagnóstico computadorizado para crianças versão IV (Lim et al., 2019); cronograma de Gantt (Lim et al., 2019); software de análises estatísticas (SAS) versões 9.3 e 9.4 (Lim et al., 2019); método de Welch e regressão multilinear stepwise (Blandón et al., 2016) biblioteca de software FMRIB para pipeline (Qian et al., 2018); software de análise funcional das neuroimagens (Qian et al., 2018); Matlab (Qian et al., 2018; Blandón et al., 2016); ferramenta Unity 3D (Faseeha et al., 2018); jogo Mind Race, que configura-se como um jogo de corridas com carros, onde a velocidade do carro aumenta com os níveis de atenção do jogador (Faseeha et al., 2018); Harvest Challenge 3D que é um jogo dividido em fases no qual para completar cada fase o jogador precisa cumprir os desafios propostos (Blandón et al., 2016); software HCI-SPT (Blandón et al., 2016); NeuroRead versão 1.1 (Blandón et al., 2016); sistema operacional Android (Arpaia et al., 2020); software aplicativo em linguagem C (Arpaia et al., 2020); software de código aberto OpenViBE (Blandón et al., 2016); filtro butterworth (Blandón et al., 2016); Elasticsearch e Middleware (Park et al., 2016).

\subsection{QP4. Quais os procedimentos que estão sendo utilizados para auxiliar no tratamento de crianças com TDAH} através de interfaces cérebro-computador?

Somente três pesquisas analisadas citam os procedimentos adotados quanto ao uso das ICCs no tratamento de crianças com TDAH. O jogo digital proposto por Blandón et al. (2016) foi desenvolvido seguindo os princípios de criação de serious games. Os pesquisadores utilizaram um sistema mindwave para detectar a atenção dos usuários. Além disso, utilizaram também o software NeuroRead para leitura dos sinais neurofisiológicos e um ambiente Matlab para processamento dos sinais. Os autores convidaram 9 crianças de uma instituição de tratamento para TDAH para avaliação do jogo e a ICC. Qian et al. (2018) e Lim et al. (2019) utilizaram o jogo Cogoland em seus testes de atenção. Associado ao jogo, as pesquisas relatas o uso do hardware head-band para transmissão dos dados neurofisiológicos, bem como, de um aparelho de ressonância magnética para análise das imagens e de questionários para avaliação dos resultados. Para avaliação do sistema proposto Lim et al. (2019) recrutaram 172 crianças diagnosticas com TDAH, atendidas em uma clínica de psiquiatria ambulatorial. E Qian et al. (2018) recrutaram 66 crianças também com TDAH atendidas em uma clínica de orientação infantil.

3.5 QP5. Quais os desafios, limitações e problemas para se utilizar interfaces cérebro-computador no tratamento de crianças com TDAH?

Os estudos inspecionados apresentaram diferenças quanto a quantidade de semanas utilizadas no tratamento, intervalo de tempo entre as sessões, tempo de duração das sessõese quantidade de crianças com TDAH avaliadas. Observou-se também que algumas crianças avaliadas apresentaram dor de cabeça, tontura, dificuldade em prestar atenção e inquietação motora, que 
podem ter relação com o desconforto do fone de ouvido, o tempo exigido para manter a atenção e manter o olhar para o monitor do computador (Lim et al., 2019). Além disso, alguns sensores utilizados para medir a atividade cerebral apresentam alta sensibilidade aos ruídos, resultando no menor nível de detecção (Faseeha et al., 2018). Os hardwares não trabalham a parte cognitiva, quanto aos softwares os jogos tem como objetivo de melhorar o nível de atenção dos participantes. Não há relatos sobre problemas para encontrar os sujeitos da amostra.

\section{Discussão}

Em relação a QP1, os estudos relatam que os benefícios e ganhos observados com o uso das ICCs no tratamento de crianças com TDAH impactam positivamente no comportamento do paciente no âmbito familiar e escolar (Blandón et al., 2016; Qian et al., 2018; Arpaia et al., 2020) possibilitando assim uma melhor socialização desse indivíduo. Para Faseeha et al. (2018) o uso de jogos atraentes associados às ICCs possibilita melhorar a atenção de indivíduos que se distraem com maior facilidade. Além disso, os jogos possibilitam também trabalhar as aptidões no campo social, bem como, o controle de suas atitudes (Park et al., 2016). Guan et al. (2020) observaram que a melhora na atençãoobtida em sua pesquisa poderá ser sustentada por um período de até 8 semanas. A redução dos sintomas de internalização com o uso das ICCs pode estar diretamente relacionada a diminuição dos sintomas de TDAH e como consequência da menor internalização tem-se a redução dos prejuízos, das experiências negativas e dos transtornos de ansiedade e humor negativo (Lim et al., 2019). Ainda no contexto dos benefícios e ganhos Guan et al. (2020) observaram que a reorganização da rede funcional do cérebro das configurações mais regulares para as mais aleatórias possibilitou a renormalização do processamento da rede de saliência.

Quanto a QP2, observou-se que diferentes hardwares foram utilizados no tratamento de crianças com TDAH por meio das ICCs. Qian et al. (2018) e Lim et al. (2019) utilizaramo head-band com sensores secos para transmitir a leitura do EEG para o computador, sendo essa transmissão realizada por bluetooth. Essa ferramenta é de grande praticidade, pois não necessita de gel condutor e permite a verificação da atividade cerebral no momento em que o paciente desempenha uma determinada função. Nos referidos estudos, o head-band foi utilizado em associação com jogos cognitivos e o efeito dessa tecnologia foi verificado por meio de imagens geradas com auxílio de um aparelho de ressonância magnética (fMRI). As imagens detectaram melhoria na conectividade funcional dos pacientes avaliados. Guan et al. (2020) também utilizaram imagens geradas pela ressonância magnética (fMRI) para avaliar as mudanças organizacionais da rede cerebral e verificaram que houve melhorias após a utilização das técnicas de treinamento de atenção nos pacientes. Verificou-se que dois estudos utilizaram o hardware Headset Mindwave Mobile da Neurosky para obtenção dos dados de EEG enquanto o paciente participava de um jogo digital (Park et al., 2016; Faseeha et al., 2018). Em Faseeha et al. (2018) o hardware em questão estava associado a uma placa Arduino Leonardo ATmega32u4 sendo a transmissão dos dados realizada via bluetooth. Já em Park et al. (2016) o hardware foi utilizado em associação com um controlador Kinect da Microsoft como uma interface de deteç̧ão de movimento sem contato com a criança. O Headset Mindwave Mobile tem como vantagens a fácil utilização (Faseeha et al., 2018) e a melhor capacidade de digitalizaçãoda atividade cerebral (Park et al., 2016). Blandón et al. (2016) também utilizaram um Headset Mindwave Mobile (sem especificação da marca) com sensores e eletrodos secos para obtenção dos dados de EEG, segundo os autores esses hardwares possibilitaram a obtenção de sinais fisiológicos gerando informações médicas valiosas para a melhor compreensão dos fenômenos do TDAH. Os hardwares utilizados em Arpaia et al. (2020) - robô Sanbot Elf humanoide, óculos de estimulação Moverio BT-200 AR, computador com placa única Raspberry Pi 3 e conversor analógico-digital Olimex EEG-SMT - possibilitaram altos níveis de envolvimento com os pacientes, impactando positivamente na eficácia do tratamento. O robô Sanbot Elf foi utilizado para desempenhar funções estabelecidas pelo paciente e os demais hardwares foram utilizados como suporte para o desenvolvimento das atividades. Todo o conjunto garantiu um feedback aos usuários em termos de movimento e fala. 
Em resposta a QP3, verificou-se o uso de vários softwares no tratamento das crianças com TDAH por meio das ICCs. Alguns softwares foram utilizados diretamente no tratamento e outros desempenharam funções de suporte. No geral, aqueles utilizados diretamente no tratamento dos pacientes com TDAH configuraram-se como jogos digitais interativos apresentando como principal objetivo o treinamento da atenção desses pacientes. Qian et al. (2018) e Lim et al. (2019) utilizaram o jogo Cogoland para treinar a atenção dos pacientes. No jogo, cada participante controla um avatar para a realização de determinadas tarefas, onde a velocidade do avatar depende do nível de atenção do jogador e quanto melhor a atenção mais rápida a atividade é realizada (Qian et al., 2018; Lim et al., 2019). Além do jogo digital, os referidos estudos utilizaram também softwares de suporte tais como: pipeline padrão da biblioteca de software FMRIB e o software de análise funcional das neuroimagens para o processamento das imagens geradas pela ressonância magnética e o Matlab para processamento de dados e construção de gráficos usados por Qian et al. (2018), bem como, o diagnóstico computadorizado para crianças usado para o diagnóstico inicial dos aspectos de atenção das crianças que participariam do tratamento, o cronograma de Gantt utilizado para elaborar o fluxograma de atividades do estudo e o software de análises estatísticas (SAS) versões 9.3 e 9.4 adotados por Lim et al. (2019). Ambos estudos relataram melhorias nos níveis de atenção após o uso do jogo digital Cogoland. Faseeha et al. (2018) fizeram uso do jogo digital Mind Race para treinar a concentração de seus pacientes. Tratase de um jogo de corrida de carros em terceira dimensão no qual o usuário necessita de concentração para jogar. Quanto maiores os níveis de atenção mais rápido o carro se movimenta. Por exercitar o cérebro para realização da atividade, o Mind Race, corroborou para melhoria do foco mental, o jogo foi desenvolvido por meio da plataforma Unity 3D, conforme Faseeha et al. (2018). O jogo digital Harvest Challenge foi utilizado por Blandón et al. (2016) e consiste em uma fazenda ecológica com atividades de aventura dividido em três fases, em cada fase o jogador deve realizar as tarefas necessárias para o prosseguimento do jogo. Adicional ao Harvest Challenge os autores usaram também os softwares HCI-SPT, NeuroRead versão 1.1, filtro butterworth e o código aberto OpenViBE para processamento dos sinais fisiológicos e biomecânicos, processamento e visualização dos sinas de EEG, processamento dos dados brutos e registro dos sinas de EEG, respectivamente. Para Blandón et al. (2016) o jogo adotado no tratamento contribuiu para o aumento dos níveis de atenção nos pacientes avaliados. Park et al. (2016) também desenvolveram um jogo digital para o treinamento comportamental por narrativas onde o jogador faz parte de uma história influenciando diretamente no desfecho da mesma, o jogo requer a atenção do participante para o desenvolvimento da história. Como softwares auxiliares os autores utilizaram o Middleware e Elasticsearch para gerenciamento dos vídeos e busca e armazenamento de dados em tempo real, respectivamente. Ainda de acordo com os autores, o jogo estabelecido ajuda as crianças a desenvolverem suas habilidades sociais enquanto aprendem a controlar o seu comportamento. Em Arpaia et al. (2020) o sistema operacional Android foi utilizado como suporte para o funcionamento do óculos Moverio BT-200 AR e do robô Sanbot Elf e o software aplicativo em linguagem C foi utilizado para o funcionamento da placa RaspberryPi 3. A Figura 2 representa o uso de ICC no tratamento de uma criança diagnosticada com TDAH. 
Figura 2. Uso de ICC em criança com TDAH.

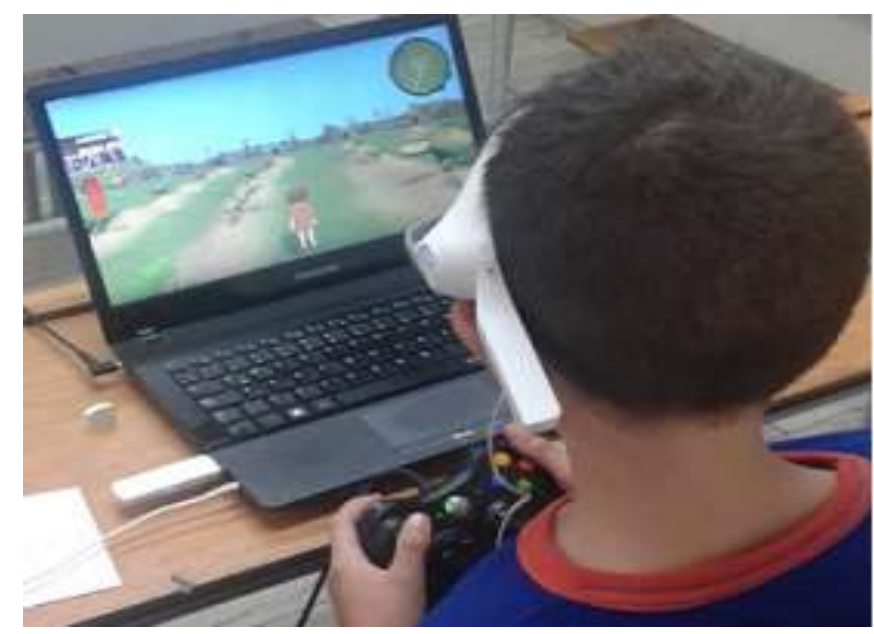

Fonte: Blandón et al. (2016).

Em relação a QP4, verificou-se que apenas três estudos relatam os procedimentos utilizados na investigação do uso das ICCs no tratamento de crianças com TDAH. Blandón et al. (2016) desenvolveram um jogo digital para treinamento da atenção aplicando os princípios de criação de serious games, devido à especificidade o design de serious games difere do design de jogos de entretenimento, porém, a pesquisa não apresenta o procedimento de criação do jogo. Observa-se ainda em Blandón et al. (2016) que para aquisição dos dados neurofisiológicos os pesquisadores utilizaram um hardware mindwave, associado a esse hardware utilizou-se o software NeuroRead para leitura dos sinais neurofisiológicos sendo esses sinais processados em ambiente Matlab. Quanto a avaliação da qualidade do jogo proposto, Blandón et al. (2016) relatam que os avaliadores foram crianças diagnosticadas com TDAH, com idades entre 5 a 12 anos e que faziam o tratamento em uma instituição local. O protocolo de avaliação consistiu um uma sessão de 30 minutos dividida em duas fases. Na primeira fase (5 minutos) as crianças passavam por um relaxamento utilizando para isso fones de ouvido e música relaxante, durante essa fase as crianças deveriam permanecer de olhos fechados. Na segunda fase (25 minutos) as crianças eram orientadas a jogar, devendo realizar as atividades em cada fase do jogo. Qian et al. (2018) e Lim et al. (2019) em seus sistemas propostos utilizaram o jogo Cogoland, porém não citam os procedimentos envolvidos na construção desse jogo. Em ambas pesquisas o hardware head-band foi utilizado para aquisição e transmissão dos dados neurofisiológicos e a eficácia da interface foi analisada por meio de imagens produzidas via ressonância magnética. Em Lim et al. (2019) as crianças recrutadas tinham idade variando entre 6 a 12 anos. Antes de participarem do jogo todas as crianças desse estudo foram submetidas a uma calibração individual por meio de uma tarefa Stroop, elaborando assim um padrão individualizado de EEG representando o estado mais atento do participante. A avaliação da qualidade ocorreu por um período de 20 semanas, com 3 sessões de treinamento por semana. Além das crianças diagnosticadas com TDAH a pesquisa utilizou também um grupo controle. Para analisar os resultados e verificar a eficácia do sistema foram utilizados uma escala de classificação de TDAH, uma lista de verificação de comportamento infantil e uma escala de classificação de eventos adversos em pediatria. Em Qian et al. (2018) os recrutados apresentaram média de idade de 9 anos e faziam tratamento no Instituto de Saúde Mental de Singapura. Os diagnósticos das crianças desse estudo foram realizados por uma equipe de psiquiatras infantis. Para determinação da amostra foi realizado teste de inteligência (Kaufman Brief Intelligence Test), sendo excluídas as crianças cujo resultado foi inferior a 70. Para avaliação da qualidade da ICC e do jogo proposto a amostra foi dividida em dois grupos no qual o primeiro grupo passou pelas intervenções e o segundo não, sendo esse utilizado como controle. Todos os participantes realizaram exames de ressonância magnética e passaram por avaliações clínicas antes de participarem do estudo. A interface e o jogo foram 
avaliados ao longo de 8 semanas, com 3 sessões de treinamento por semana durando 30 minutos cada. Para avaliação dos resultados foram utilizados questionários neuropsicológicos.

Quanto a QP5 é possível considerar que o uso das ICCs no tratamento de pacientes com TDAH possui desafios a serem vencidos, uma vez que, os artigos inspecionados apresentam divergências em vários aspectos metodológicos, demonstrando que ainda não existe um padrão de utilização dessa ferramenta para esse tipo de tratamento. Essas divergências podem se configurar como limitações ao uso das ICCs no tratamento de TDAH, pois dificultam a realização de uma análise comparativa entre os estudos para verificar se as respostas encontradas estão condizentes. Os efeitos adversos relatados por alguns participantes e descritos em Lim et al. (2019) também configuram-se como desafios a serem vencidos, os mesmos podem estar associados a metodologia aplicada. A necessidade de um ambiente silencioso para utilização dos sensores de EEG relatado em Faseeha et al. (2018) também pode ser compreendido como uma limitação da tecnologia aplicada. $\mathrm{O}$ fato de a maior parte dos estudos não citar a técnica utilizada para avaliação dos aspectos de atenção e cognitivos pode ser considerado um dos maiores problemas encontrados, uma vez que, dificulta a replicação do estudo.

\section{Conclusão}

Com base no que foi exposto, pode-se afirmar que, no geral, a utilização das ICCs corrobora com o tratamento de crianças com TDAH apresentando resultados positivos. A utilização dessa tecnologia possibilitou melhorias nos aspectos de atenção e controle comportamental do indivíduo, refletindo beneficamente na sua relação familiar e social. Pode-se considerar que mais pesquisas nesse contexto são necessárias para que se possa mitigar os desafios, limitações e problemas encontrados. Nesse sentido, sugere-se que os estudos futuros investiguem principalmente os aspectos metodológicos de uso das ICCs no tratamento de pacientes com TDAH para que seja possível a determinação de uma metodologia de referência para a utilização dessa ferramenta.

\section{Referências}

American Psychiatric Association. Manual diagnóstico e estatístico de transtornos mentais, DSM-5. 5.ed. Porto Alegre: Artmed, 2014. http://www.niip.com.br/wp-content/uploads/2018/06/Manual-Diagnosico-e-Estatistico-de-Transtornos-Mentais-DSM-5-1-pdf.pdf.

Abrahão, N. S. \& Fantacini, R. A. F. (2017). Transtorno do déficit de atenção com hiperatividade (TDAH): desafios e possibilidade frente a sala de aula. Research, Society and Development, 6(3), 222-236. DOI: https://doi.org/10.17648/rsd-v6i3.159.

Arpaia, P., Duraccio, L., Moccaldi, N. \& Rossi, S. (2020). Wearable brain-computer interface instrumentation for robot-based rehabilitation by augmented reality. IEEE Transactions on Instrumentation and Measurement, 69(9), 6362-6371. DOI: https://doi.org/10.1109/TIM.2020.2970846.

Blandón, D. Z., Muñoz, J. E., Lopez, D. S. \& Gallo, O. H. (2016). Influence of a BCI neurofeedback videogame in children with ADHD. Quantifying the brain activity through a EEG signal processing dedicado toolbox. Colombian Computing Conference (CCC), Popayan, Colômbia, 11. DOI: 10.1109 / ColumbianCC.2016.7750788.

Boland, H., Disalvo, M., Fried, R., Woodworth, K. Y., Wilens, T., Faraone, S. V. \& Biederman, J. (2020). A literature review and meta-analysis on the effectsof ADHD medications on functional outcomes. Journal of Psychiatric Research, 123, 21-30. DOI: https://doi.org/10.1016/j.jpsychires.2020.01.006.

Cortese, S. \& Coghill, D. (2018). Twenty years of research on attention-deficit/hyperactivitydisorder (ADHD): looking back, looking forward. Evid Based Mental Health, 21(4), 173-176. DOI: https://doi.org/10.1136/ebmental-2018-300050.

Dickstein, S. G., Bannon. K., Castellanos, F. X. \& Milham, M. P. (2006). The neural correlates of attention deficit hyperactivity disorder: an ALE metaanalysis. Journal of Child Psychology and Psychiatry, 47(10), 1051-1062. DOI: https://doi.org/10.1111/j.1469-7610.2006.01671.x.

Faseeha, U., Naseem, M., Saleem, J., Jahan, A. \& Jamil, N. (2018). Virtual gaming. International Conference on Mathematics, Actuarial Science, Computer Science and Statistics (MACS), Karachi, Paquistão, 12. DOI: 10.1109/MACS.2018.8628437.

Guan, C., Lim, C. G., Fung, D., Zhou, H. J., Krishnan, R. \& Lee, T. S. (2020). BCI facilitates the improvement of cognitive functions in children and elderly. International Winter Conference on Brain-Computer Interface (BCI), Gangwon, Coreia (Sul), 8. DOI: 10.1109 / BCI48061.2020.9061625.

Geppert, S. E., Huster, R. J. \& Herrmann, C. S. (2017). EEG-neurofeedback as a tool to modulate cognition and behavior: a review tutorial. Frontiers in Human Neuroscience,11(51), 1-19. DOI: https://doi.org/10.3389/fnhum.

Hechtman, L., Swanson, J. M., Sibley, M. H., Stehli, A., Owens, E. B., Mitchell, J. T., Arnold, L. E., Molina, B. S. G., Hinshaw, S. P., Jensen, P. S., Abikoff, H. B., Algorta, G. P., Howard, A. L., Hoza, B., Etcovitch, J., Houssais, S., Lakes, K. D. \& Nichols, J. Q. (2016). Functional adult outcomes 16 years after 
childhood diagnosis of attention-deficit/hyperactivity disorder: mta results. Journal of the american academy of child and adolescent psychiatry, 55(11), 945-952. DOI: https://doi.org/10.1016/j.jaac.2016.07.774.

Jiang, Y., Abiri, R. \& Zhao, X. (2017). Tuning up the old brain with new tricks: attention training via neurofeedback. Frontiers in Aging Neuroscience, 9(52), 1-9. DOI: https://doi.org/10.3389/fnagi.2017.00052.

Kessler, R. C., Adler, L., Barkley, R., Biederman, J., Conners, C. K., Demler, O., Faraone, S. V., Greenhill, L. L., Howes, M. J., Secnik, K., Spencer, T., Ustun, T. B., Walters, E. E. \& Aslavsky, A. M. (2006). The prevalence and correlates of adult ADHD in the United States: results from the national comorbiditysurvey replication. The American Journal of Psychiatry, 163(4), 716-723. DOI: https://doi.org/10.1176/ajp.2006.163.4.716.

Kitchenham, B. (2004). Procedures for performing systematic reviews. Keele, UK, Keele University, 33(2004), 1-26. Disponível em: https://www.inf.ufsc.br/ aldo.vw/kitchenham.pdf.

Lim, C. G., Poh, X. W. W., Fung, S. S. D., Guan, C., Bautista, D., Cheung, Y. B., Zhang, H., Yeo, S. N., Krishnan, R. \& Lee, T. S. (2019). A randomized controlled trial of a brain-computer interface based attention training program for ADHD. Plos One, 14(15), e0216225. DOI: https://doi.org/10.1371/journal.pone.0216225.

Marçal, E., Kubrusly, M. \& Silva, C. L. O. (2017). Avaliando aplicações móveis para o ensino em saúde: uma revisão sistemática. Revista Tecnologia Educacional, 217 ,

https://www.researchgate.net/publication/332118391_avaliando_aplicacoes_moveis_para_o_ensino_em_saude_uma_revisao_sistematica.

Munakata, Y., Michaelson, L., Barker, J. \& Chevalier, N. (2013). As funções executivas na infância. In: MORTON, J. B. (Org.). Enciclopédia sobre o desenvolvimento na primeira infância. Ontario: CEECD. https://www.enciclopedia-crianca.com/sites/default/files/dossiers-complets/pt-pt/funcoesexecutivas.pdf.

Park, K., Kihl, T., Park, S., Kim, M. \& Chang, E. J. (2016). Narratives and sensor driven cognitive behavior training game platform. International Conference onSoftware Engineering Research, Management and Applications (SERA), Towson, MD, EUA, 14. DOI: 10.1109 / SERA.2016.7516137.

Qian, X., Loo, B. R. Y., Castellanos, F. X., Liu, S., Koh, H. L., Poh, X. W. W., Krishnan, R., Fung, D., Chee, M. W. L., Guan, C., Lee, T. S., Lim, C. G. \& Zhou, J. (2018). Brain-computer-interface-based intervention re-normalizes brain functionalnetwork topology in children with attention deficit/hyperactivity disorder. Translational Psychiatry, 8(149), 1-11. DOI: https://doi.org/10.1038/s41398-018-0213-8.

Rief, S. F. (Ed.). (2005). How to reach and teach children with ADD / ADHD: practical techniques, strategies and interventions. $2^{\text {a }}$. ed. San Francisco: Josseybass.

Silva, A. B. B. (Ed.). (2014). Mentes inquietas: TDAH: Desatenção, hiperatividade e impulsividade. $4^{\text {a }}$. ed. São Paulo: Globo.

Snyder, S. M. \& Hall, J. R. (2006). A meta-analysis of quantitative eeg power associated with attention-deficit hyperactivity disorder. Journal of Clinical Neurophysiology, 23(5), 441-456. DOI: https://doi.org/10.1097/01.wnp.0000221363.12503.78. 